\title{
Rational and Exponential Legendre Tau Method on Steady Flow of a Third Grade Fluid in a Porous Half Space
}

\author{
F. Baharifard ${ }^{1}$ - Saeed Kazem ${ }^{2}$ - K. Parand ${ }^{3}$
}

Published online: 16 September 2015

(C) Springer India Pvt. Ltd. 2015

\begin{abstract}
In this paper, we decide to compare rational and exponential Legendre functions Tau approach to solve the governing equations for the flow of a third grade fluid in a porous half space. Firstly, we estimate an upper bound for function approximation based on mentioned functions in semi-infinite domain, and discuss that the analytical functions have a superlinear convergence for these basis. Also the operational matrices of derivative and product of these functions are presented to reduce the solution of this problem to the solution of a system of nonlinear algebraic equations. The comparison of the results of rational and exponential Legendre Tau methods with numerical solution shows the efficiency and accuracy of these methods. We also make a comparison between these two methods themselves and show that using exponential functions, leads to more accurate results and faster convergence in this problem.
\end{abstract}

Keywords Rational Legendre · Exponential Legendre · Function approximation · Tau method · Third grade fluid · Porous half space

Mathematics Subject Classifications 34B15 · 34B40

\section{Introduction}

In recent years, there has been some interest on flows of non-Newtonian fluids where inertial effects are significant. Many materials such as polymer solutions or melts, drilling muds,

Saeed Kazem

saeedkazem@gmail.com; saeedkazem@aut.ac.ir

1 School of Computer Science, Institute for Research in Fundamental Sciences (IPM), Tehran, Iran

2 Department of Applied Mathematics, Faculty of Mathematics and Computer Science, Amirkabir University of Technology, No. 424, Hafez Ave, Tehran 15914, Iran

3 Department of Computer Sciences, Faculty of Mathematical Sciences, Shahid Beheshti University, Evin, Tehran 19839, Iran 
clastomers, certain oils and greases and many other emulsions are classified as non-Newtonian fluids. The fluids of second and third grade have been studied successfully in various types of flow situations which form a subclass of the fluids of the differential type. The third grade fluid models even for steady flow exhibits such characteristics. Due to the widespread applications such as oil recovery, paper and textile coating, composite manufacturing processes, mixture theory, filtration processes, geothermal engineering and insulation systems, flow through porous media received substantial attention $[1,2]$.

The present study deals with the problem of non-Newtonian fluid of third grade in a porous half space [3]. For unidirectional flow, Hayat et al. [3] have generalized the relation

$$
(\nabla p)_{x}=-\frac{\mu \varphi}{k}\left(1+\frac{\alpha_{1}}{\mu} \frac{\partial}{\partial t}\right) u,
$$

for a second grade fluid to the following modified Darcy's Law for a third grade fluid

$$
(\nabla p)_{x}=-\frac{\varphi}{k}\left[\mu u+\alpha_{1} \frac{\partial u}{\partial t}+2 \beta_{3}\left(\frac{\partial u}{\partial y}\right)^{2} u\right],
$$

where $u$ denotes the fluid velocity, $\mu$ is the dynamic viscosity and $p$ is the pressure. $k$ and $\varphi$ represent the permeability and porosity of the porous half space which occupies the region $y>0$, respectively $\alpha_{1}$ and $\beta_{3}$ are material constants. Defining non-dimensional fluid velocity $f$ and the coordinate $z$

$$
\begin{aligned}
& z=\frac{V_{0}}{v} y, \quad f(z)=\frac{u}{V_{0}}, \\
& V_{0}=u(0), \quad v=\frac{\mu}{\rho},
\end{aligned}
$$

where $v$ and $V_{0}$ represent the kinematic viscosity and the boundary value problem, modeling the steady state flow of a third grade fluid in a porous half space, becomes as below such as prime denotes differentiation with respect to $z$ [3]:

$$
\begin{aligned}
& f^{\prime \prime}(z)+b_{1} f^{\prime}(z)^{2} f^{\prime \prime}(z)-b_{2} f(z) f^{\prime}(z)^{2}-c f(z)=0, \\
& f(0)=1, \lim _{z \rightarrow \infty} f(z)=0,
\end{aligned}
$$

where $b_{1}, b_{2}$ and $c$ are defined as:

$$
b_{1}=\frac{6 \beta_{3} V_{0}^{4}}{\mu v^{2}}, \quad b_{2}=\frac{2 \beta_{3} \varphi V_{0}^{2}}{k \mu}, \quad c=\frac{\varphi v^{2}}{k V_{0}^{2}} .
$$

Above parameters are depended, whereof

$$
b_{2}=\frac{b_{1} c}{3} \text {. }
$$

Therefore, we can rewrite Eq. (1) as

$$
f^{\prime \prime}(z)+b_{1} f^{\prime}(z)^{2} f^{\prime \prime}(z)-\frac{b_{1} c}{3} f(z) f^{\prime}(z)^{2}-c f(z)=0 .
$$

The homotopy analysis method for solution of Eq. (1) is found in [3]. Later Ahmad [4] gave the asymptotic form of the solution and utilize this information to develop a series solution. Recently, authors in [5] solved this problem by radial basis functions.

One of the powerful method for solving the ordinary differential equations (ODEs) is spectral method in both terms of accuracy and simplicity. Spectral methods, in the context of 
numerical schemes for differential equations, generically belong to the family of weighted residual methods (WRMs) [6]. WRMs represent a particular group of approximation techniques, in which the residuals (or errors) are minimized in a certain way and thereby leading to specific methods including Galerkin, Petrov-Galerkin, collocation and Tau formulations [7-13].

The basis of spectral method is always polynomials (orthogonal polynomials). These types of basis for approximation of enough smooth functions in finite domain leads to the exponential convergence. Many science and engineering problems arise in unbounded domains. For example the steady flow of a third grade fluid in a porous half space (Eq. (3)). The orthogonal polynomials such as Legendre, Chebyshev, Hermite and Laguerre polynomials can not satisfied the steady property of this type of fluid $f(\infty)=$ constant. Therefore, a number of spectral methods for treating unbounded domains have been proposed by different researchers.

Guo $[14,15]$ proposed a method that proceeds by mapping the original problem in an unbounded domain to a problem in a bounded domain, and then using suitable Jacobi polynomials to approximate the resulting problems.

There is another effective direct approach for solving such problems is based on rational approximations. Christov [16] and Boyd [17,18] developed some spectral methods on unbounded intervals by using mutually orthogonal systems of rational functions. Boyd [17] defined a new spectral basis, named rational Chebyshev functions on the semi-infinite interval, by mapping to the Chebyshev polynomials. Guo et al. [19] introduced a new set of rational Legendre functions which are mutually orthogonal in $L^{2}(0,+\infty)$. They applied a spectral scheme using the rational Legendre functions for solving the Korteweg-de Vries equation on the half-line. Boyd et al. [20] applied pseudospectral methods on a semi-infinite interval and compared rational Chebyshev, Laguerre and mapped Fourier sine methods.

Authors of [21-23], applied spectral method to solve nonlinear ordinary differential equations on semi-infinite intervals. Their approach was based on rational Tau and collocation methods. In Tau method, they obtained the operational matrices of derivative and product of rational Chebyshev and Legendre functions and then applied these matrices together to reduce the solution of these problems to the solution of a system of algebraic equations. The Tau approach is an approximation technique introduced by Lonczos [24] in 1938 to solve differential equations. Tau method is based on expanding the required approximate solution as the elements of a complete set of orthogonal functions. This method may be viewed as a special case of the so-called Petrov-Galerkin method. But, unlike the Galerkin approximation, the expansion functions are not required to satisfy the boundary constraint individually [25-30].

In the current paper, our main aim is to apply the Tau method with two basis functions on the semi-infinite interval for solving the steady flow of a third grade fluid in a porous half space and compare the results of them together. Also any discussion on the error analysis of approximation of the function defined in $[0, \infty)$ by rational and exponential Legendre functions have been investigated. In the next section, we obtain an upper bound for estimating the error of function approximation by these basis functions. And then we show that the error of these approximation is superlinear convergence.

The remainder of this paper is organized as follows: "Properties of Rational And Exponential Legendre Functions" Section reviews the desirable properties of two functions that obtain by mapping the Legendre polynomials, namely rational Legendre and exponential Legendre functions. In "Application of the Methods" Section, we apply Tau method with two mentioned functions as basis functions separately to solve the problem. we compare our results together and with numerical solutions in "Results and Discussion" Section. Finally, "Conclusions" Section makes concluding remarks. 


\section{Properties of Rational and Exponential Legendre Functions}

This section is devoted to the introduction of rational and exponential Legendre functions, that both of them are defined on the semi-infinite interval. Then, the operational matrices of derivative and their product are given.

\section{Rational Legendre Functions}

The Legendre polynomials are orthogonal in the interval $[-1,1]$ with respect to the weight function $\rho(y)=1$. They can be determined by the following recurrence formula [31]:

$$
\begin{aligned}
& P_{0}(y)=1, \quad P_{1}(y)=y, \\
& P_{n+1}(y)=\left(\frac{2 n+1}{n+1}\right) y P_{n}(y)-\left(\frac{n}{n+1}\right) P_{n-1}(y), \quad n \geq 1 .
\end{aligned}
$$

The following Legendre polynomials property can be helped our discussion in the "Function Approximation" Section;

$$
\int_{-1}^{1} y^{m} P_{n}(y) d y= \begin{cases}0, & m<n, \\ \frac{2^{n+1} n !^{2}}{(2 n+1) !}, & n=m .\end{cases}
$$

The rational Legendre functions, is denoted by $R_{n}(x)=P_{n}\left(\frac{x-L}{x+L}\right)$, where $L$ is a constant parameter and sets the length scale of the mapping. Boyd [32] has offered some guidelines for optimizing the map parameter $L$.

$R_{n}(x)$ satisfies in the following recurrence relation:

$$
\begin{aligned}
& R_{0}(x)=1, \quad R_{1}(x)=\frac{x-L}{x+L} \\
& R_{n+1}(x)=\left(\frac{2 n+1}{n+1}\right)\left(\frac{x-L}{x+L}\right) R_{n}(x)-\left(\frac{n}{n+1}\right) R_{n-1}(x), \quad n \geq 1 .
\end{aligned}
$$

By using above recursive formula the first four rational Legendre functions are obtained as below:

$$
\left\{\begin{array}{l}
R_{0}(x)=1, \\
R_{1}(x)=\frac{x-L}{x+L}, \\
R_{2}(x)=\frac{3}{2}\left(\frac{x-L}{x+L}\right)^{2}-\frac{1}{2}, \\
R_{3}(x)=\frac{5}{2}\left(\frac{x-L}{x+L}\right)^{3}-\frac{3}{2}\left(\frac{x-L}{x+L}\right) .
\end{array}\right.
$$

In Fig. 1 the behavior of these four functions for $L=1$ are plotted.

Rational Legendre functions (RL) are orthogonal with respect to the weight function $w_{r}(x)=\frac{2 L}{(x+L)^{2}}$ in the interval $[0,+\infty)$, with the orthogonality property:

$$
\int_{0}^{+\infty} R_{n}(x) R_{m}(x) w_{r}(x) d x=\frac{2}{2 n+1} \delta_{n m},
$$

where $\delta_{n m}$ is the Kronecker function. 


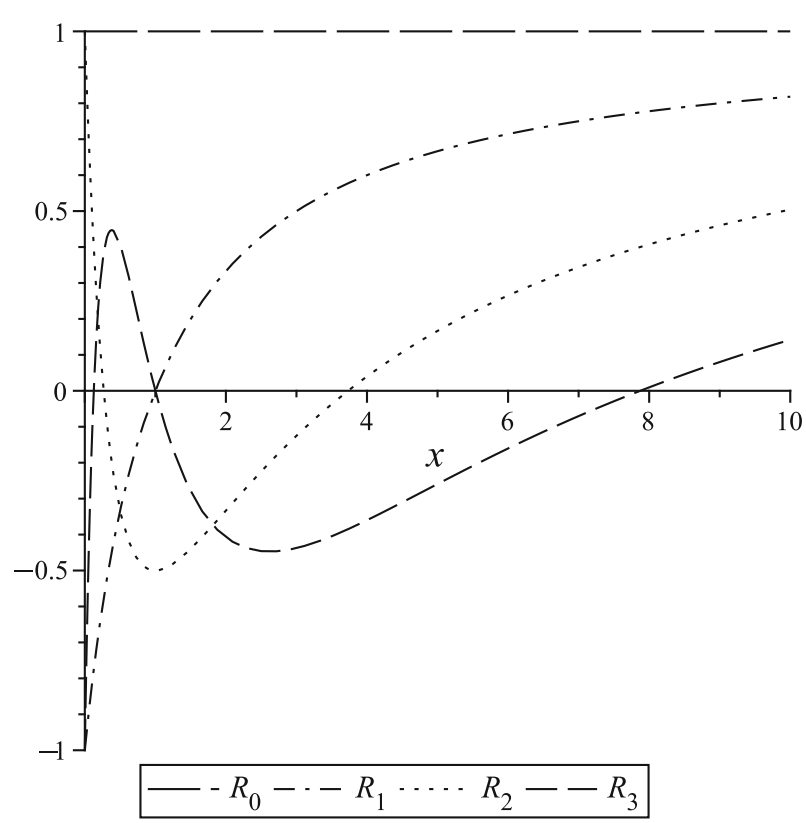

Fig. 1 Graph of four rational Legendre functions for $L=1$

\section{Exponential Legendre Functions}

Exclusive of rational functions we can use exponential transformation to have new functions which are also defined on the semi-infinite interval. The exponential Legendre (EL) functions can be defined by $E_{n}(x)=P_{n}(1-2 \exp (-x / L))$, where parameter $L$ is a constant parameter and, like rational functions, it sets the length scale of the mapping.

$E_{n}(x)$ satisfies in the following recurrence relation:

$$
\begin{aligned}
& E_{0}(x)=1, \quad E_{1}(x)=1-2 \exp (-x / L), \\
& E_{n+1}(x)=\left(\frac{2 n+1}{n+1}\right)(1-2 \exp (-x / L)) E_{n}(x)-\left(\frac{n}{n+1}\right) E_{n-1}(x), \quad n \geq 1 .
\end{aligned}
$$

By using above recursive formula the first four exponential Legendre functions are obtained as below:

$$
\left\{\begin{array}{l}
E_{0}(x)=1 \\
E_{1}(x)=1-2 \exp (-x / L) \\
E_{2}(x)=\frac{3}{2}[1-2 \exp (-x / L)]^{2}-\frac{1}{2} \\
E_{3}(x)=\frac{5}{2}[1-2 \exp (-x / L)]^{3}-\frac{3}{2}[1-2 \exp (-x / L)]
\end{array}\right.
$$

In Fig. 2 the behavior of these four functions for $L=1$ are plotted. 


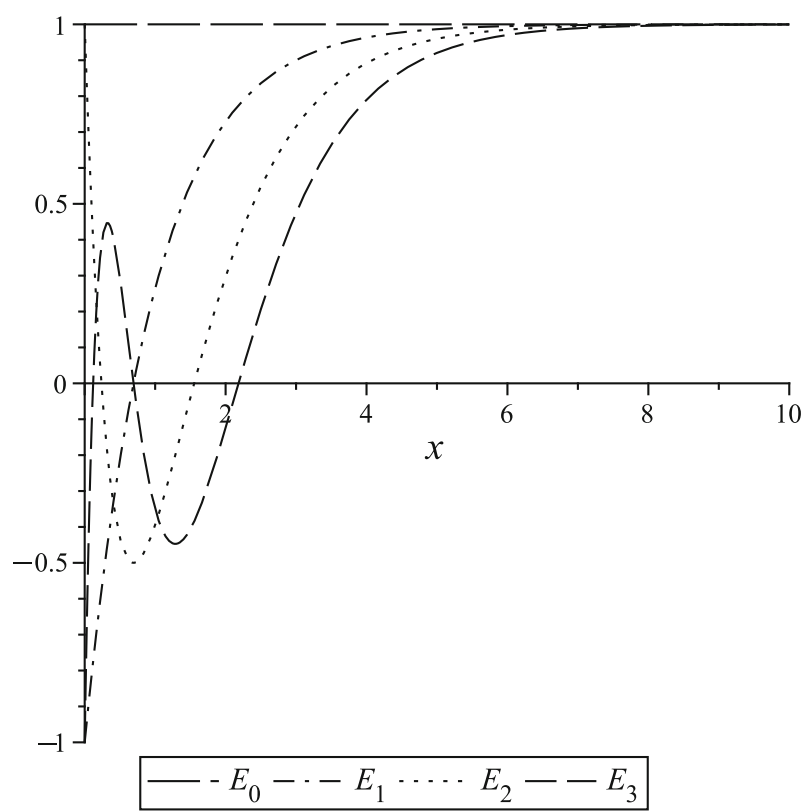

Fig. 2 Graph of four exponential Legendre functions for $L=1$

Exponential Legendre functions are orthogonal with respect to the weight function $w_{e}(x)=\frac{2}{L} \exp (-x / L)$ in the interval $[0,+\infty)$, with the orthogonality property, the same as Eq. (8):

$$
\int_{0}^{+\infty} E_{n}(x) E_{m}(x) w_{e}(x) d x=\frac{2}{2 n+1} \delta_{n m} .
$$

\section{Function Approximation}

Let $w(x)$ is $w_{r}(x)$ or $w_{e}(x)$ denotes a non-negative, integrable, real-valued function over the interval $\Lambda=[0, \infty)$. We define

$$
L_{w}^{2}(\Lambda)=\left\{v: \Lambda \longrightarrow \mathbb{R} \mid v \text { is measurable and }\|v\|_{w}<\infty\right\},
$$

where

$$
\|v\|_{w}^{2}=\int_{0}^{+\infty} v^{2}(x) w(x) d x
$$

is the norm induced by the inner product of the space $L_{w}^{2}(\Lambda)$,

$$
\langle u, v\rangle_{w}=\int_{0}^{+\infty} u(x) v(x) w(x) d x .
$$


Thus $\left\{\phi_{j}(x)\right\}_{j \geq 0}$, are considered $\left\{R_{j}(x)\right\}_{j \geq 0}$ or $\left\{E_{j}(x)\right\}_{j \geq 0}$, denotes a system which is mutually orthogonal under (12), i.e.,

$$
\left\langle\phi_{n}(x), \phi_{m}(x)\right\rangle_{w}=\frac{2}{2 n+1} \delta_{n m} .
$$

The classical Weierstrass theorem implies that such a system is complete in the space $L_{w}^{2}(\Lambda)$. Thus, for any function $f(x) \in L_{w}^{2}(\Lambda)$ the following expansion holds

$$
f(x)=\sum_{j=0}^{+\infty} a_{j} \phi_{j}(x)
$$

where

$$
a_{j}=\frac{2 j+1}{2} \int_{0}^{+\infty} \phi_{j}(x) f(x) w(x) d x
$$

If $f(x)$ in Eq. (13) is truncated up to the $N$ th terms, then it can be written as

$$
f(x) \simeq f_{N}(x)=\sum_{j=0}^{N-1} a_{j} \phi_{j}(x)=A^{T} \phi(x)
$$

with

$$
\begin{aligned}
& A=\left[a_{0}, a_{1}, \ldots, a_{N-1}\right]^{T}, \\
& \phi(x)=R(x)=\left[R_{0}(x), R_{1}(x), \ldots, R_{N-1}(x)\right]^{T} \\
& \quad \text { or } \\
& \phi(x)=E(x)=\left[E_{0}(x), E_{1}(x), \ldots, E_{N-1}(x)\right]^{T} .
\end{aligned}
$$

Now, we can estimate an upper bound for function approximation in a special case. In first, the error may be defined in the following form

$$
e_{n}=\left\|f(x)-f_{n}(x)\right\|_{w}^{2} .
$$

The completeness of the system $\left\{\phi_{i}(x)\right\}_{i \geq 0}$ is equivalent to the following property

$$
f_{n}(x) \longrightarrow f(x), \quad e_{n} \longrightarrow 0 \quad \text { as } \quad n \longrightarrow \infty \text {. }
$$

Lemma 1 The error which is defined in (18) can be rewritten as

$$
e_{n}=\sum_{i=n}^{\infty} \frac{2 i+1}{2}\left\langle f(x), \phi_{i}(x)\right\rangle_{w}^{2} .
$$

Proof The completeness of the system $\left\{\phi_{i}(x)\right\}_{i \geq 0}$ helped us to consider the error as

$$
e_{n}=\left\|\sum_{i=n}^{\infty} a_{i} \phi_{i}(x)\right\|_{w}^{2} \text {. }
$$


Using the definition of $\|\cdot\|_{w}$, one has

$$
e_{n}=\sum_{i=n}^{\infty} \sum_{j=n}^{\infty} a_{i} a_{j}\left\langle\phi_{i}(x), \phi_{j}(x)\right\rangle_{w}=\sum_{i=n}^{\infty} \sum_{j=n}^{\infty} a_{i} a_{j} \frac{2}{2 i+1} \delta_{i j}=\sum_{i=n}^{\infty} \frac{2 a_{i}^{2}}{2 i+1} .
$$

And using Eq. (14) the Lemma can be proved.

This Lemma shows that the convergence rate is involved with function $f(x)$. Now, by knowing that the function $f(x) \in L_{w}^{2}(\Lambda)$ have some good properties, we could present an upper bound for estimating the error of function approximation by this basis function.

Theorem 1 Let $f_{n}(x)$ is function approximation of $f(x) \in L_{w}^{2}(\Lambda)$ obtained by (15) and $\mathcal{F}(y)=f(\Psi(y))$ is analytic on $[-1,1]$, then an error bound for this approximation can be presented as follows:

$$
e_{n} \leq \sum_{i=n}^{\infty} \frac{4^{i+1} M_{i}^{2} i !^{2}}{2(2 i) !(2 i+1) !}
$$

where

$$
\Psi(y)=L \frac{1+y}{1-y} \quad \text { or } \quad-L \ln \left(\frac{1-y}{2}\right),
$$

and $M_{i}=\operatorname{Max}\left|\mathcal{F}^{(i)}(y)\right|, y \in(-1,1)$.

Proof We have the following properties for each case of $\Psi(y)$,

Case 1: $\Psi(y)=L \frac{1+y}{1-y}, \quad R_{n}(\Psi(y))=P_{n}(y)$,

$$
w_{r}(\Psi(y))=\frac{(1-y)^{2}}{2 L}, \quad d x=\frac{2 L}{(1-y)^{2}} d y,
$$

Case 2: $\quad \Psi(y)=L \ln \left(\frac{2}{1-y}\right), \quad E_{n}(\Psi(y))=P_{n}(y)$,

$$
w_{e}(\Psi(y))=\frac{1-y}{L}, \quad d x=\frac{L}{1-y} d y .
$$

By substituting each case in $\left\langle f(x), \phi_{i}(x)\right\rangle_{w}$, one has

$$
\left\langle f(x), \phi_{i}(x)\right\rangle_{w}=\int_{-1}^{1} \mathcal{F}(y) P_{i}(y) d y .
$$

Also knowing that $\mathcal{F}(y)$ is analytic, we have

$$
\left\langle f(x), \phi_{i}(x)\right\rangle_{w}=\sum_{j=0}^{i-1} \frac{\mathcal{F}^{(j)}(0)}{j !} \int_{-1}^{1} y^{j} P_{i}(y) d y+\frac{\mathcal{F}^{(i)}\left(\xi_{i}\right)}{i !} \int_{-1}^{1} y^{i} P_{i}(y) d y, \quad \xi_{i} \in(-1,1) .
$$

Using the Legendre polynomials property mentioned in Eq. (5), the above equation can be rewritten in the following form

$$
\left\langle f(x), \phi_{i}(x)\right\rangle_{w}=\frac{\mathcal{F}^{(i)}\left(\xi_{i}\right)}{i !} \frac{2^{i+1}(i !)^{2}}{(2 i+1) !} \leq \frac{2^{i+1} i ! M_{i}}{(2 i+1) !} .
$$

The theorem can be proved by substituting above inequality in Eq. (19). 
It may be understood from the previous theorem that any function defined in $L_{w}^{2}(\Lambda)$, which their mapping under transformation $L \frac{1+y}{1-y}$ or $L \ln \left(\frac{2}{1-y}\right)$ are analytic, has a series solution in the form (15) corresponds to a exponential rate of convergence. But with more discussions, we could get the better result. The next theorem would show that the error defined in Eq. (18) have superlinear convergence. Firstly, we define the order of convergence is called superlinear. Among the several definitions, we choose the following one

Definition $1 x_{n} \longrightarrow \bar{x}$ with superlinear convergence if there is a positive sequence $\lambda_{n} \longrightarrow 0$ and an integer number $N$ such that

$$
\left|x_{n+1}-\bar{x}\right| \leq \lambda_{n}\left|x_{n}-\bar{x}\right|, \quad n \geq N .
$$

Theorem 2 In Theorem 1, let $M \geq M_{i}$, then the error is superlinear convergence to zero.

Proof Using Theorem 1. we have

$$
e_{n} \leq M^{2} \sum_{i=n}^{\infty} \frac{4^{i+1} i !^{2}}{2(2 i) !(2 i+1) !}=M^{2} \sum_{i=n}^{\infty} \frac{4^{i+1} i ! i !}{2(2 i) ! !(2 i-1) ! !(2 i+1) ! !(2 i) ! !},
$$

where $(2 i-1) ! !=(2 i-1) \times(2 i-3) \times \cdots \times 3 \times 1$ and $(2 i) ! !=2 i \times(2 i-2) \times \cdots \times 4 \times 2=2^{i} i !$. Then one has

$$
e_{n} \leq 2 M^{2} \sum_{i=n}^{\infty} \frac{1}{(2 i-1) ! !(2 i+1) ! !} \leq 2 M^{2} \sum_{i=n}^{\infty} \frac{1}{(2 i) !} .
$$

We define $x_{n}=\sum_{i=n}^{\infty} 1 /(2 i)$ !, and then, there is a positive sequence

$$
\lambda_{n}=1-\frac{1}{(2 n !) \sum_{i=n}^{\infty} \frac{1}{(2 i) !}} \longrightarrow 0
$$

that $\left|x_{n+1}\right| \leq \lambda_{n}\left|x_{n}\right|$. Therefore, $x_{n}$ and subsequently $e_{n}$ are superlinear convergence to zero.

According to Theorem 2, any function defined in $L_{w}^{2}([0, \infty))$, which their mapping under transformation $L \frac{1+y}{1-y}$ or $L \ln \left(\frac{2}{1-y}\right)$ are analytic, has a series solution in the form (15) with the superlinear convergence.

\section{Operational Matrix of Derivative}

\section{Derivative Matrix for Rational Legendre Functions}

The derivative of the vector $R(x)$ defined in Eq. (16) can be expressed as

$$
R^{\prime}(x)=\frac{d R}{d x} \simeq D_{r} R(x),
$$

where $D_{r}$ is the $N \times N$ operational matrix for the derivative. Differentiating Eq. (6), we have:

$$
\begin{aligned}
& R_{0}^{\prime}(x)=0 \\
& R_{1}^{\prime}(x)=\frac{1}{L}\left[\frac{2}{3} R_{0}(x)-R_{1}(x)+\frac{1}{3} R_{2}(x)\right], \\
& R_{n+1}^{\prime}(x)=\frac{2 n+1}{n+1}\left(R_{n}(x) \cdot R_{1}(x)\right)^{\prime}-\frac{n}{n+1} R_{n-1}^{\prime}(x), \quad n \geq 1 .
\end{aligned}
$$


The matrix $D_{r}$ can be calculated by using Eq. (22). Authors of [21] obtained that this matrix is a lower?-Hessenberg matrix and can be expressed as $D_{r}=\frac{1}{L}\left(D_{1}+D_{2}\right)$; where $D_{1}$ is a tridiagonal matrix with below form:

$$
D_{1}=\operatorname{diag}\left(\frac{7 i^{2}-i-2}{2(2 i+1)},-i, \frac{i(i+1)}{2(2 i+1)}\right), \quad i=0, \ldots, N-1,
$$

and the $d_{i j}$ elements of matrix $D_{2}$ are obtained from

$$
d_{i j}= \begin{cases}0, & j \geq i-1 \\ (-1)^{i+j+1}(2 j+1), & j<i-1 .\end{cases}
$$

For $N=6$, we have

$$
D_{r}=\frac{1}{L}\left(\begin{array}{rrrrrr}
0 & 0 & 0 & 0 & 0 & 0 \\
\frac{2}{3} & -1 & \frac{1}{3} & 0 & 0 & 0 \\
-1 & \frac{12}{5} & -2 & \frac{3}{5} & 0 & 0 \\
1 & -3 & \frac{29}{7} & -3 & \frac{6}{7} & 0 \\
-1 & 3 & -5 & \frac{53}{9} & -4 & \frac{10}{9} \\
1 & -3 & 5 & -7 & \frac{84}{11} & -5
\end{array}\right)
$$

\section{Derivative Matrix for Exponential Legendre Functions}

The derivative of the vector $E(x)$ defined in Eq. (17) can be expressed as

$$
E^{\prime}(x)=\frac{d E}{d x}=D_{e} E(x)
$$

where $D_{e}$ is the $N \times N$ operational matrix for the derivative. Differentiating the Eq. (9), we get:

$$
\begin{aligned}
& E_{0}^{\prime}(x)=0, \\
& E_{1}^{\prime}(x)=\frac{1}{L}\left[E_{0}(x)-E_{1}(x)\right], \\
& E_{n+1}^{\prime}(x)=\frac{2 n+1}{n+1}\left(E_{n}(x) \cdot E_{1}(x)\right)^{\prime}-\frac{n}{n+1} E_{n-1}^{\prime}(x), \quad n \geq 1 .
\end{aligned}
$$

By using Eq. (25), the matrix $D_{e}$ can be generated. The matrix $D_{e}$ is a lower-triangular matrix and can be expressed as $D_{e}=\frac{1}{L}\left(\widehat{D_{1}}+\widehat{D_{2}}\right)$; where $\widehat{D_{1}}$ is a diagonal matrix which is obtained from

$$
\widehat{D_{1}}=\operatorname{diag}(-i), \quad i=0, \ldots, N-1,
$$

and $\widehat{D_{2}}$ is exactly equal to $D_{2}$, Eq. (23).

for $N=6$, we have

$$
D_{e}=\frac{1}{L}\left(\begin{array}{rrrrrr}
0 & 0 & 0 & 0 & 0 & 0 \\
1 & -1 & 0 & 0 & 0 & 0 \\
-1 & 3 & -2 & 0 & 0 & 0 \\
1 & -3 & 5 & -3 & 0 & 0 \\
-1 & 3 & -5 & 7 & -4 & 0 \\
1 & -3 & 5 & -7 & 9 & -5
\end{array}\right)
$$

As it can be seen, the form of above matrix is simpler than that of the derivation matrix for $R(x)$. In addition, $D_{e}$ is a lower-triangular matrix but $D_{r}$ is a lower-Hessenberg matrix, 
so more zero elements exists in $D_{e}$. Consequently, it can be computed faster. In the other hand, the truncated derivative matrix of $D_{e}$ exactly satisfies Eq. (24), but $D_{r}$ doesn't have this property. Therefore, the accuracy of the exponential Legendre functions is better to approximate the function derivatives in $[0, \infty)$.

\section{The Product Operational Matrix}

\section{Product Matrix for Rational Legendre Functions}

The following property of the product of two rational Legendre function vectors will also be applied:

$$
R(x) R^{T}(x) A \simeq \tilde{A} R(x),
$$

where $\tilde{A}$ is an $N \times N$ product operational matrix for the vector $A$. Using Eq. (26) and by the orthogonal property Eq. (8), the elements $\tilde{A}_{i j},(i=0, \ldots, N-1, j=0, \ldots, N-1)$ of the matrix $\tilde{A}$ can be calculated from

$$
\tilde{A}_{i j}=\left(j+\frac{1}{2}\right) \sum_{k=0}^{N-1} a_{k} g_{i j k}
$$

where $g_{i j k}$ is given by

$$
g_{i j k}=\int_{0}^{+\infty} R_{i}(x) R_{j}(x) R_{k}(x) w_{r}(x) d x .
$$

Also, the product of two rational Legendre functions, $R_{i}(x)$ and $R_{j}(x)$, can be approximated as below [33]:

$$
R_{i}(x) R_{j}(x)=\sum_{l=0}^{j} \frac{d_{j-l} d_{l} d_{i-l}}{d_{i+j-l}}\left(\frac{2 i+2 j-4 l+1}{2 i+2 j-2 l+1}\right) R_{i+j-2 l}(x),
$$

where $d_{l}$ is obtained by the below formula

$$
d_{l}=\frac{(2 l) !}{2^{l}(l !)^{2}}, \quad j<i .
$$

Multiplying Eq. (28) by $R_{k}(x) w_{r}(x)$ integrating from 0 to $+\infty$ and using the orthogonal property, we have

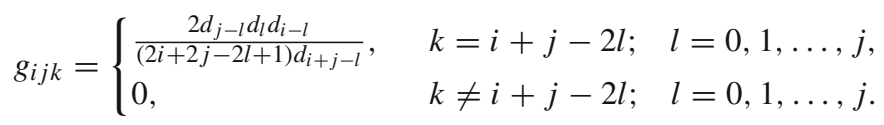

\section{Product Matrix for Exponential Legendre Functions}

For the product of the two exponential Legendre function vectors we also have:

$$
E(x) E^{T}(x) A \simeq \tilde{A} E(x),
$$

in which the elements $\tilde{A}_{i j}$ of the matrix $\tilde{A}$ are obtained similar to Eq. (27), such as $g_{i j k}$ is computed the same as Eq. (29). 


\section{Application of the Methods}

In this part, we apply the Tau method with rational Legendre and exponential Legendre functions as basis functions for solving the steady flow of a third grade fluid in a porous half space, Eq. (3), with initial conditions of Eq. (1).

We consider $\phi(z)=R(z)$ in below formulas to apply rational Legendre Tau method (RLT) and assume $\phi(z)=E(z)$ for using exponential Legendre Tau method (ELT) and approximate the problem.

We express $f(z)$ and $f^{(j)}(z)$ as below

$$
\begin{aligned}
& f(z) \simeq f_{N}(z)=\sum_{i=0}^{N-1} a_{i} \phi_{i}(z)=A^{T} \phi(z), \\
& f^{(j)}(z) \simeq f_{N}^{(j)}(z)=\sum_{i=0}^{N-1} a_{i} \phi_{i}^{(j)}(z) \simeq A^{T} D^{j} \phi(z) \quad j=1,2,3,
\end{aligned}
$$

where $D^{j}$ is the $j$ th power of the matrix $D_{r}$ or $D_{e}$ given in Eqs. (21) or (24).

By using Eqs. (31) and (32), we have

$$
\begin{aligned}
f_{N}^{\prime}(z)^{2} f_{N}^{\prime \prime}(z) & \simeq A^{T} D \phi(z) \phi^{T}(z) D^{T} A \phi^{T}(z)\left(D^{2}\right)^{T} A \\
& =A^{T} D \phi(z) \phi^{T}(z) U \phi^{T}(z) V=A^{T} D \tilde{U} \tilde{V} \phi(z), \\
f_{N}(z) f_{N}^{\prime}(z)^{2} & \simeq A^{T} \phi(z) \phi^{T}(z) D^{T} A \phi^{T}(z) D^{T} A \\
& =A^{T} \phi(z) \phi^{T}(z) U \phi^{T}(z) U=A^{T} \tilde{U} \tilde{U} \phi(z),
\end{aligned}
$$

where $U=D^{T} A, V=\left(D^{2}\right)^{T} A$ and the matrices $\tilde{U}$ and $\tilde{V}$ can be calculated similarly to Eqs. (26) or (30).

The functions $M(z)$ for Eq. (3), can be constructed by using Eqs. (31-34). So we have:

$$
M(z)=\left[A^{T} D^{2}+b_{1} A^{T} D \tilde{U} \tilde{V}-\frac{b_{1} c}{3} A^{T} \tilde{U} \tilde{U}-c A^{T}\right] \phi(z) .
$$

Similar to the typical Tau method $[26,34]$ we generate $(N-2)$ algebraic equations by applying

$$
\left\langle M(z), \phi_{k}(z)\right\rangle=\int_{0}^{+\infty} M(z) \phi_{k}(z) w(z) d z=0, \quad k=0,1, \ldots, N-3,
$$

where $w(z)$ is $w_{r}(z)$ or $w_{e}(z)$.

Using Eq. (31) for the boundary conditions in Eq. (1) and we have:

$$
f_{N}(0)=A^{T} \phi(0)=1, \quad \lim _{z \rightarrow+\infty} f_{N}(z)=\lim _{z \rightarrow+\infty} A^{T} \phi(z)=0 .
$$

Equations (35) and (3) generate a set of $N$ nonlinear algebraic equations. Consequently, the unknown coefficients $a_{i}$ 's of the vector $A$ in Eq. (31) can be calculated.

\section{Results and Discussion}

In this section, the numerical results of the model equation presented in Eq. (3), obtained by using RLT and ELT methods. In this problem, the first derivative at zero $\left(f^{\prime}(0)\right)$ is an important point for the numerical solution. In Table 1 , the resulting values of $f^{\prime}(0)$ that 
Table 1 Comparison $f^{\prime}(0)$ of problem obtained by RLT, ELT, with shooting method solution by various parameters $b_{1}$ and $c$

\begin{tabular}{lllll}
\hline$b_{1}$ & $c$ & Shooting method & RLT & ELT \\
\hline 0.3 & 0.5 & -0.691280 & -0.691493 & -0.691279 \\
0.6 & & -0.678301 & -0.678511 & -0.678302 \\
0.9 & & -0.667327 & -0.667528 & -0.667327 \\
1.2 & & -0.657838 & -0.658029 & -0.657837 \\
0.6 & 0.3 & -0.533303 & -0.533545 & -0.533302 \\
& 0.6 & -0.738008 & -0.738116 & -0.738007 \\
& 0.9 & -0.887467 & -0.887350 & -0.887467 \\
& 1.2 & -1.008653 & -1.008516 & -1.008653 \\
\hline
\end{tabular}

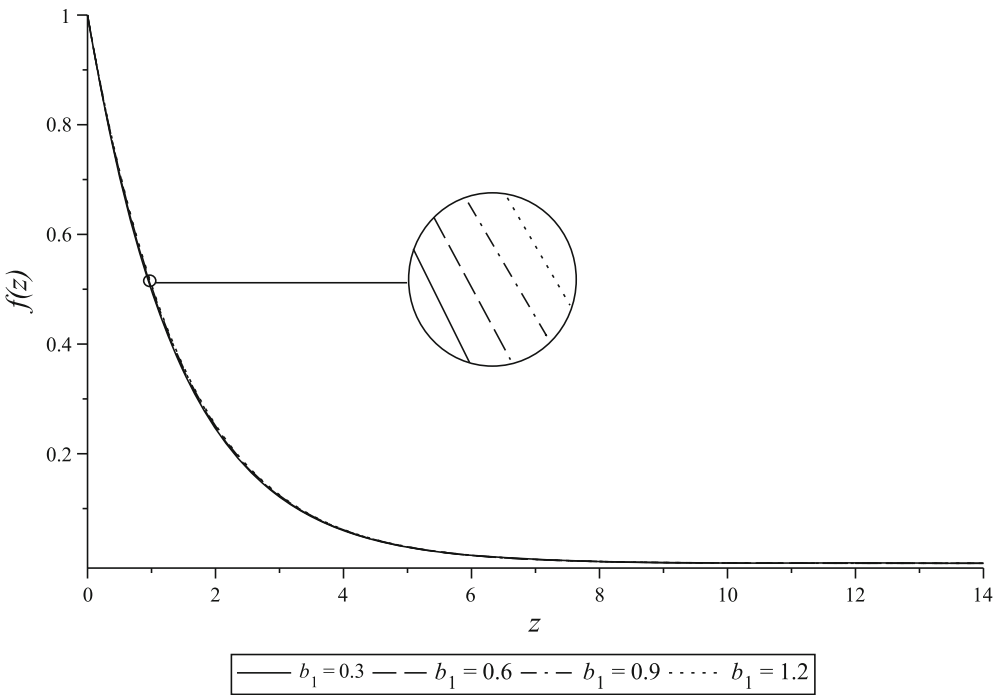

Fig. 3 Variation of the velocity distribution for the various values of $b_{1}$ and $c=0.5$ obtained by rational Legendre Tau method

obtained by using the RLT and ELT methods with $N=10$ and $L=4$ are presented for some various values of $b_{1}$ and $c$. In this Table we made comparison between present methods and numerical solutions which are obtained by shooting method. The Table shows a good agreement between two present methods and numerical solutions. But, as can be seen, ELT method gives us results with higher degree of accuracy than RLT method. By rational functions, we could reach to the same results of accuracy as ELT method if $N$ be considered more than 17. So using exponential Legendre functions, provide an effective but simple way to improve the convergence of the solution by Tau method. Also, this Table shows that for constant value of $c$, an increase in $b_{1}$ leads to a rise in $b_{2}$ (by using Eq. (2)), $f^{\prime}(0)$ and the velocity. This result is obviously appeared in Figs. 3 and 4. In the other hand, with constant value of $b_{1}$ and the increase in $c, f^{\prime}(0)$ and the velocity decrease. Subsequently, Figs. 5 and 6 represent the velocity distribution for the various values of the parameter $c$. Therefore, increasing or decreasing the value of $b_{2}$ has no effect on velocity.

Tables 2 and 3 show the comparison of results for $f(z)$ obtained by two proposed methods and the numerical solution where $b_{1}=0.6$ and $c=0.5$ and 0.9 , respectively. The relative 


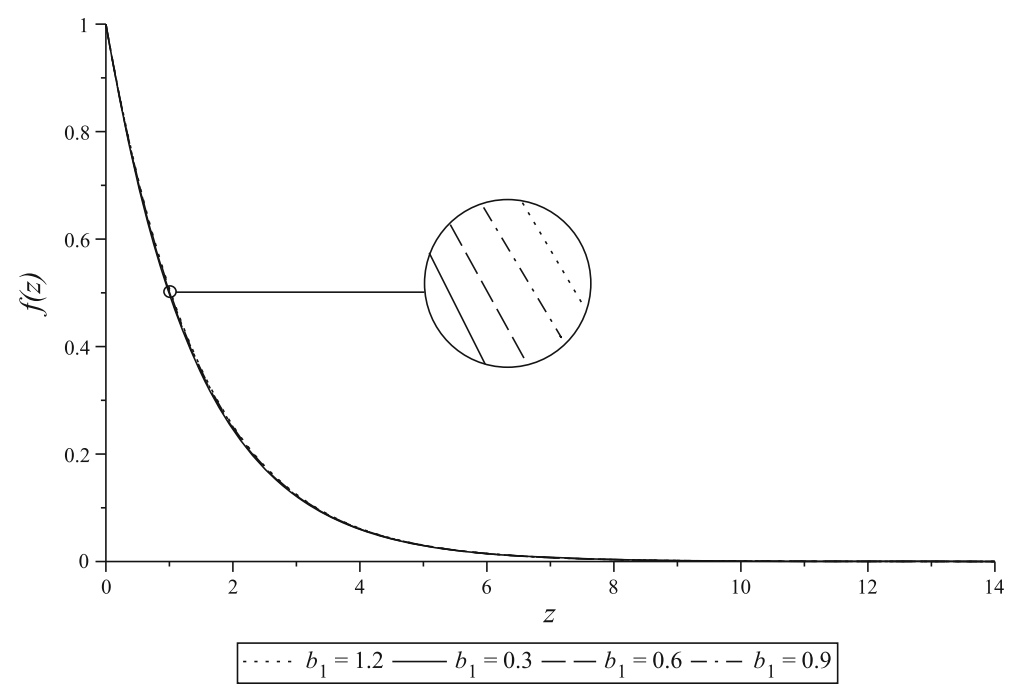

Fig. 4 Variation of the velocity distribution for the various values of $b_{1}$ and $c=0.5$ obtained by exponential Legendre Tau method

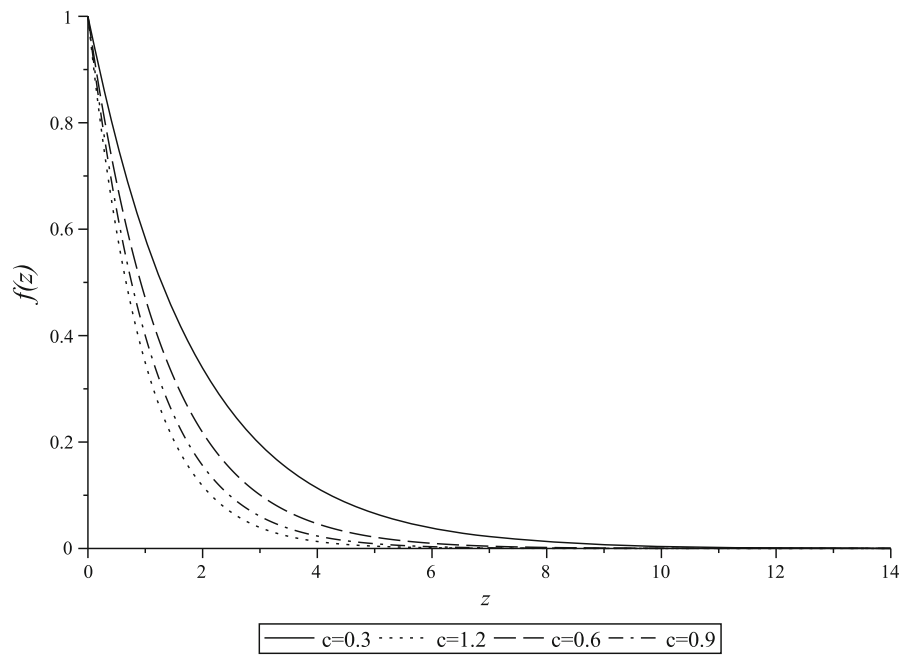

Fig. 5 Variation of the velocity distribution for the various values of $c$ and $b_{1}=0.6$ obtained by rational Legendre Tau method

errors in these Tables show that by using exponential Legendre functions, we get higher accurate solution than using rational functions for approximate this problem.

Logarithmic graphs of absolute coefficients $\left|a_{i}\right|$ of RLT and ELT functions in the approximate solutions for $b_{1}=0.6$ and $c=0.5$ and 0.9 , are shown in Figs. 7 and 8, respectively. These graphs illustrate that the methods have an appropriate convergence rate by small $N$.

To show the accuracy of this method and give a comparison between rational and exponential Legendre Tau methods, we define the residual functions by using Eq. (3) as

$$
\operatorname{Res}(z)=f_{N}^{\prime \prime}(z)+b_{1} f_{N}^{\prime}(z)^{2} f_{N}^{\prime \prime}(z)-b_{2} f_{N}(z) f_{N}^{\prime}(z)^{2}-c f_{N}(z) .
$$




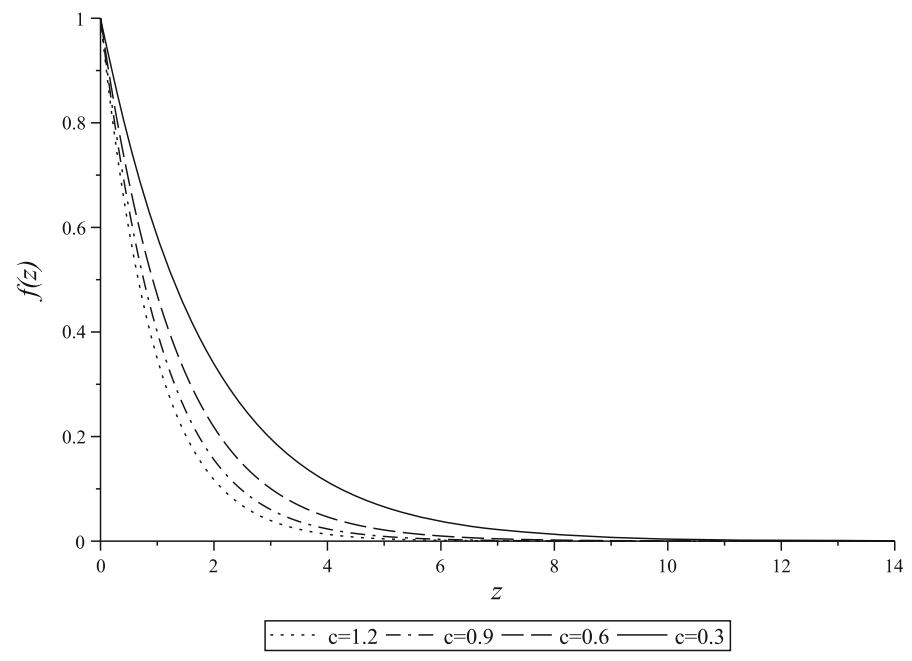

Fig. 6 Variation of the velocity distribution for the various values of $c$ and $b_{1}=0.6$ obtained by exponential Legendre Tau method

Table 2 Comparison of $f(z)$ for problem, between RLT, ELT and Runge-Kutta solution when $b_{1}=0.6$ and $c=0.5$ with $N=10$

\begin{tabular}{llllll}
\hline$z$ & Runge-Kutta & RLT & Relative error & ELT & Relative error \\
\hline 0.0 & 1.00000 & 1.00000 & 0.00000 & 1.00000 & 0.00000 \\
0.2 & 0.87261 & 0.87261 & 0.00000 & 0.87261 & 0.00000 \\
0.4 & 0.76063 & 0.76063 & 0.00000 & 0.76063 & 0.00000 \\
0.6 & 0.66243 & 0.66243 & 0.00000 & 0.66243 & 0.00000 \\
0.8 & 0.57650 & 0.57650 & 0.00000 & 0.57650 & 0.00000 \\
1.0 & 0.50144 & 0.50143 & 0.00001 & 0.50144 & 0.00000 \\
1.2 & 0.43595 & 0.43595 & 0.00000 & 0.43595 & 0.00000 \\
1.6 & 0.32920 & 0.32920 & 0.00000 & 0.32920 & 0.00000 \\
2.0 & 0.24839 & 0.24837 & 0.00002 & 0.24838 & 0.00001 \\
2.5 & 0.17455 & 0.17455 & 0.00000 & 0.17455 & 0.00000 \\
3.0 & 0.12262 & 0.12264 & 0.00002 & 0.12261 & 0.00001 \\
3.5 & 0.08612 & 0.08617 & 0.00005 & 0.08611 & 0.00001 \\
4.0 & 0.06048 & 0.06054 & 0.00006 & 0.06047 & 0.00001 \\
4.5 & 0.04248 & 0.04252 & 0.00004 & 0.04247 & 0.00001 \\
5.0 & 0.02984 & 0.02984 & 0.00000 & 0.02982 & 0.00002 \\
\hline
\end{tabular}

Now by defining the Eduction norm of $\operatorname{Res}(z)$ and knowing that $\operatorname{Res}(z) \in \mathbf{R L}^{M}$ or $\mathbf{E L}^{M}$, where the maximum value of $M=3 N+1$ and

$$
\begin{aligned}
& \boldsymbol{R} \boldsymbol{L}^{M}=\operatorname{span}\left\{R_{0}(z), R_{1}(z), \ldots, R_{M}(z)\right\}, \\
& \boldsymbol{E} \boldsymbol{L}^{M}=\operatorname{span}\left\{E_{0}(z), E_{1}(z), \ldots, E_{M}(z)\right\} .
\end{aligned}
$$


Table 3 Comparison of $f(z)$ for problem, between RLT, ELT and Runge-Kutta solution when $b_{1}=0.6$ and $c=0.9$ with $N=10$

\begin{tabular}{llllll}
\hline$z$ & Runge-Kutta & RLT & Relative error & ELT & Relative error \\
\hline 0.0 & 1.00000 & 1.00000 & 0.00000 & 1.00000 & 0.00000 \\
0.2 & 0.83610 & 0.83609 & 0.00001 & 0.83610 & 0.00000 \\
0.4 & 0.69720 & 0.69721 & 0.00001 & 0.69720 & 0.00000 \\
0.6 & 0.58014 & 0.58016 & 0.00002 & 0.58014 & 0.00000 \\
0.8 & 0.48195 & 0.48196 & 0.00001 & 0.48195 & 0.00000 \\
1.0 & 0.39987 & 0.39987 & 0.00000 & 0.39987 & 0.00000 \\
1.2 & 0.33148 & 0.33145 & 0.00003 & 0.33148 & 0.00000 \\
1.6 & 0.22738 & 0.22733 & 0.00005 & 0.22738 & 0.00000 \\
2.0 & 0.15577 & 0.15575 & 0.00002 & 0.15577 & 0.00000 \\
2.5 & 0.09700 & 0.09706 & 0.00006 & 0.09700 & 0.00000 \\
3.0 & 0.06038 & 0.06050 & 0.00012 & 0.06038 & 0.00000 \\
3.5 & 0.03758 & 0.03770 & 0.00012 & 0.03757 & 0.00001 \\
4.0 & 0.02338 & 0.02345 & 0.00007 & 0.02338 & 0.00000 \\
4.5 & 0.01455 & 0.01454 & 0.00001 & 0.01455 & 0.00000 \\
5.0 & 0.00905 & 0.00896 & 0.00009 & 0.00906 & 0.00001 \\
\hline & & & & & \\
\hline
\end{tabular}

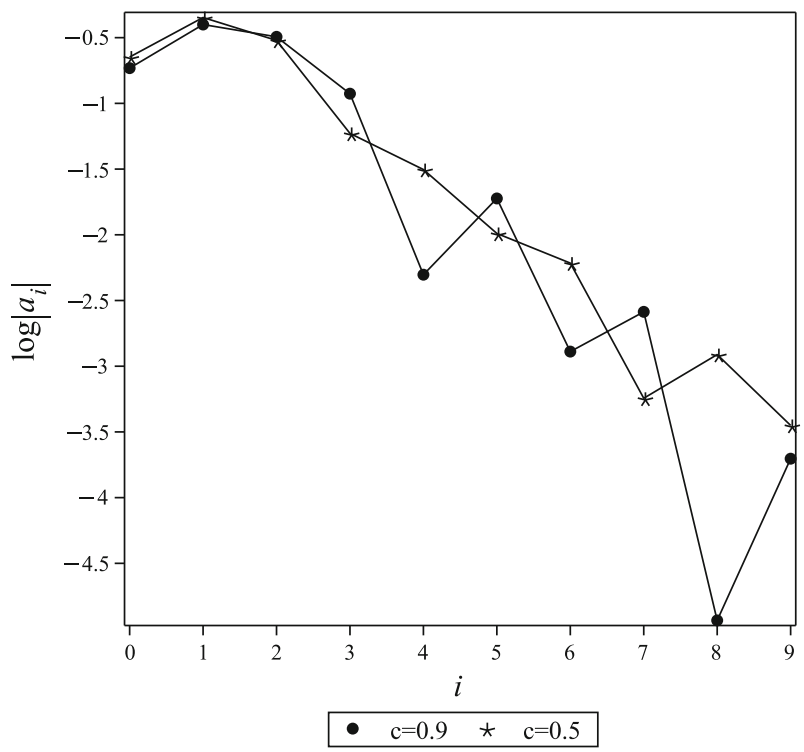

Fig. 7 Logarithmic graph of absolute coefficients $\left|a_{i}\right|$ of the rational Legendre functions when $b_{1}=0.6$

We can determine $\|\operatorname{Res}\|_{w}^{2}$ by the well-known Gauss quadrature formulation in the forms of

$$
\|\operatorname{Res}\|_{w}^{2}=\int_{0}^{\infty} \operatorname{Res}^{2}(z) w(z) d z=\sum_{i=0}^{M} w_{i} \operatorname{Res}^{2}\left(z_{i}\right),
$$




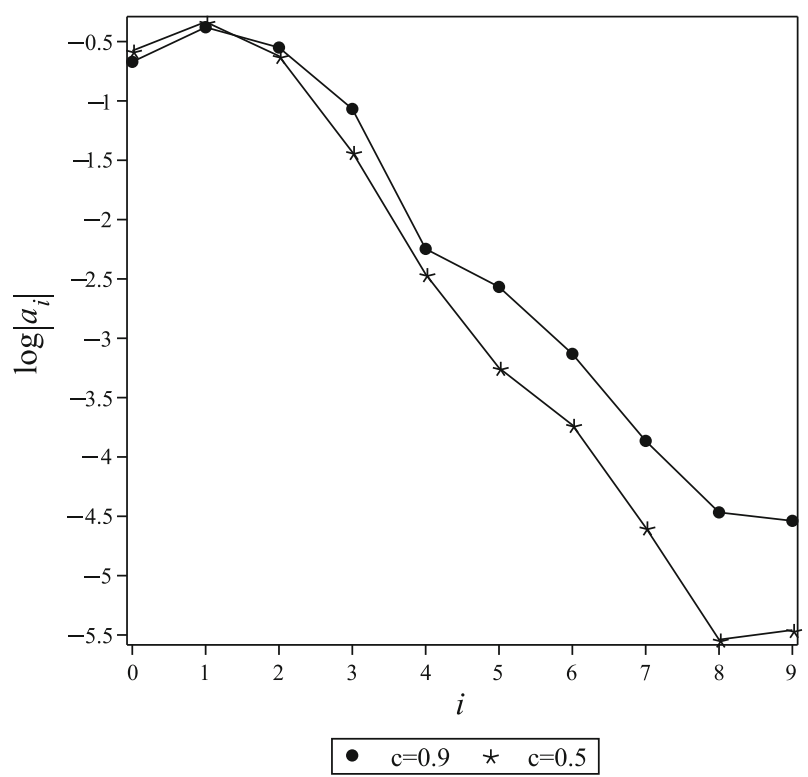

Fig. 8 Logarithmic graph of absolute coefficients $\left|a_{i}\right|$ of the exponential Legendre functions when $b_{1}=0.6$

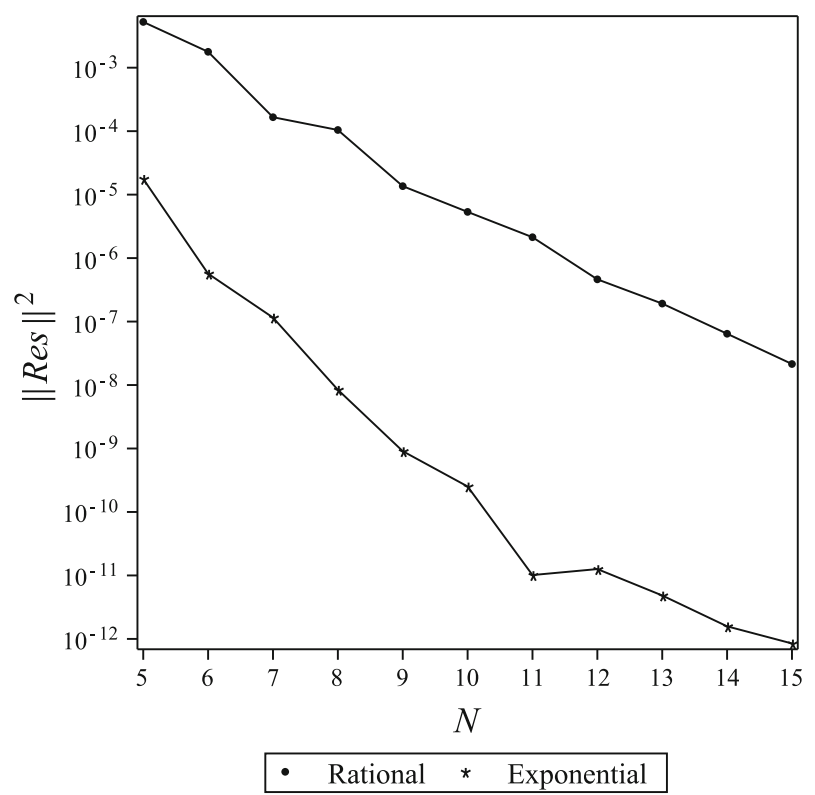

Fig. 9 Graph of $\|$ Res $\|^{2}$ by RLT and ELT methods when $b_{1}=0.6$ and $c=0.5$

$$
\begin{aligned}
& z_{i}=L \frac{s_{i}+1}{1-s_{i}}, \quad w_{i}=\frac{2 L}{z_{i}\left(z_{i}+L\right)^{2}\left(R_{M+1}^{\prime}\left(z_{i}\right)\right)^{2}}, \quad \text { Rational Legendre, } \\
& z_{i}=-L \ln \left(\frac{1-s_{i}}{2}\right), \quad w_{i}=\frac{2}{L^{2}\left(\mathrm{e}^{\frac{z_{\mathrm{i}}}{\mathrm{L}}}-1\right)\left(\mathrm{E}_{\mathrm{M}+1}^{\prime}\left(\mathrm{z}_{\mathrm{i}}\right)\right)^{2}}, \quad \text { Exponential Legendre }
\end{aligned}
$$




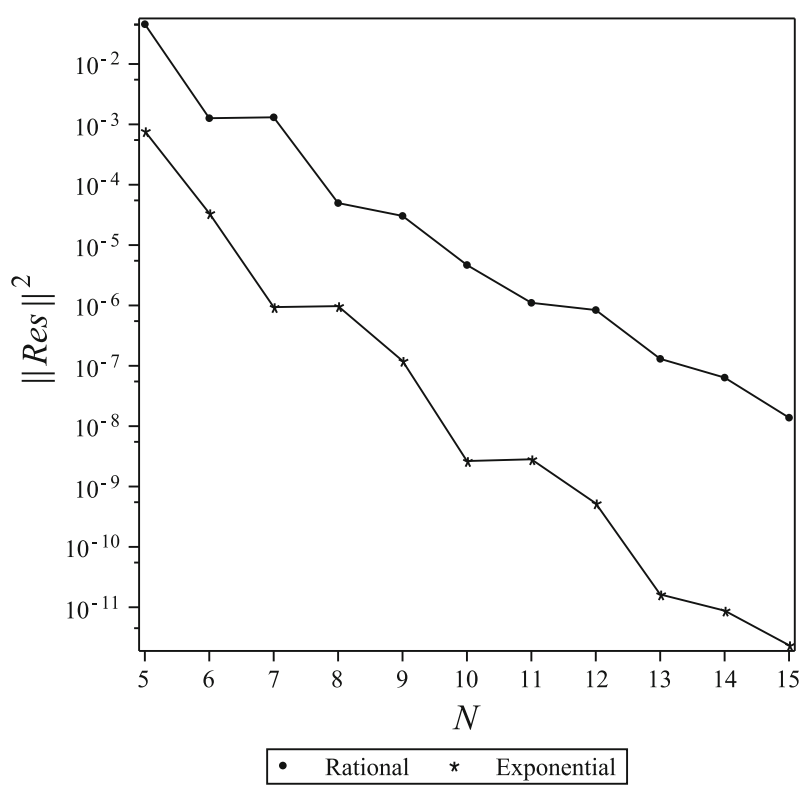

Fig. 10 Graph of $\|$ Res $\|^{2}$ by RLT and ELT methods when $b_{1}=0.6$ and $c=0.9$

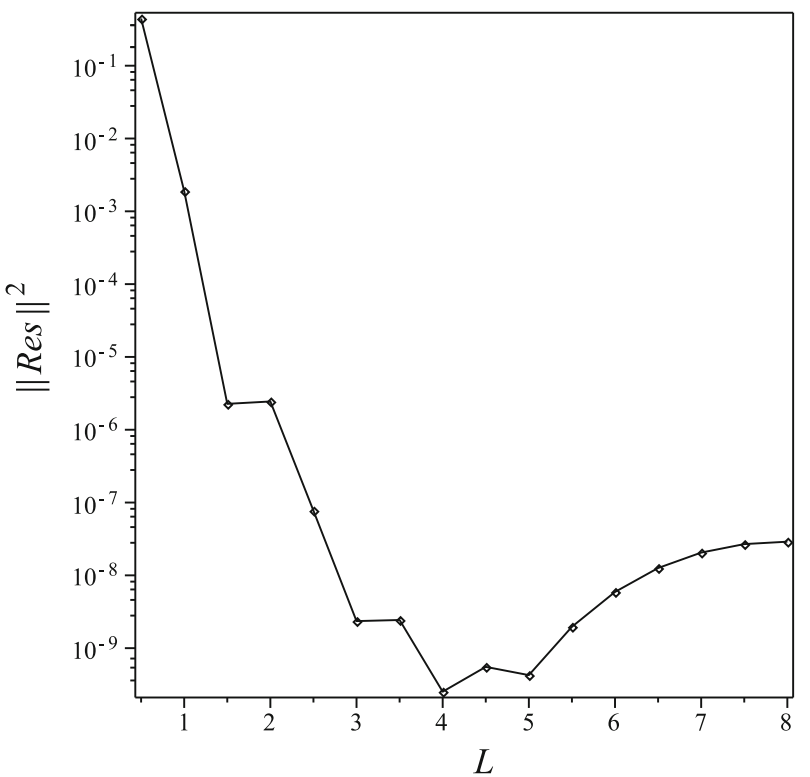

Fig. 11 Graph of $\|\operatorname{Res}\|^{2}$ of ELT methods by $N=10$ and various $L$ when $b_{1}=0.6$ and $c=0.5$

and $s_{i}$ are zero of $M+1$-order of Legendre polynomial.

Figures 9 and 10 show the logarithmic graphs of the $\|\operatorname{Res}\|_{w}^{2}$ at $b_{1}=0.6$ and $c=0.5$ and 0.9 for rational and exponential Legendre Tau methods. These graphs illustrate the accuracy of the methods. Furthermore, they show that the accuracy of the ELT method is better than 


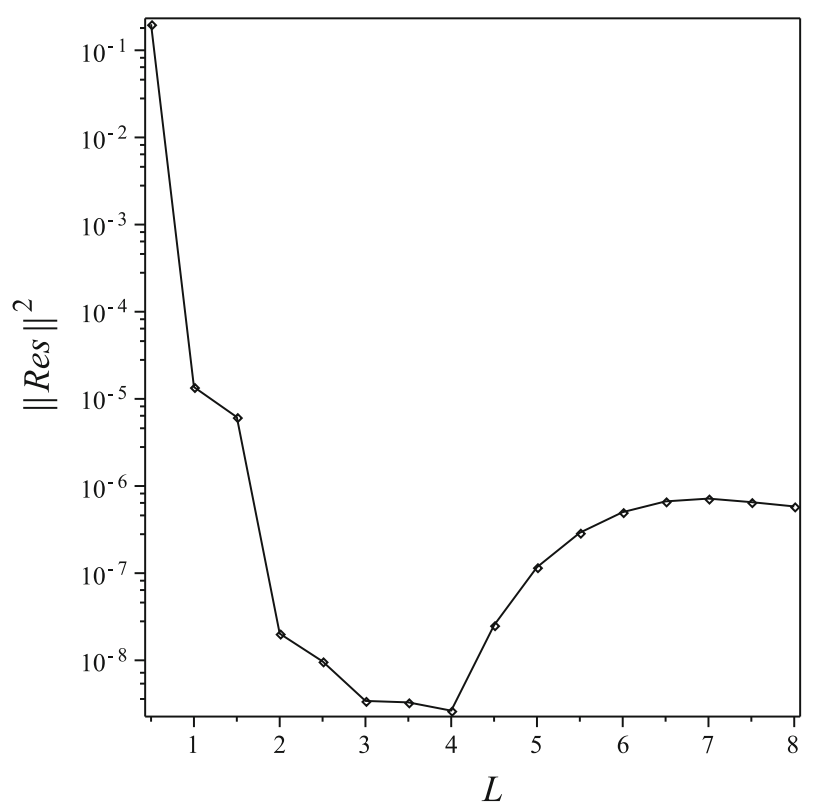

Fig. 12 Graph of $\|\operatorname{Res}\|^{2}$ of ELT methods by $N=10$ and various $L$ when $b_{1}=0.6$ and $c=0.9$

ELT method in this problem. Also in Figs. 11 and 12 the logarithmic graphs of the $\|$ Res $\|_{w}^{2}$ of ELT methods by $N=10$ and various values of $L$ are shown. These figures illustrate the interval that we can choose for $L$ parameter to get applicable result. That is why we select $L=4$ for solving this problem.

\section{Conclusions}

In present study, the steady flow of the third grade fluid in a porous half space, is considered. Based on modified Darcys law, the flow over a suddenly moved flat plate is discussed numerically by using the operational matrices of derivative and product of rational and exponential Legendre functions together with the Tau method. The influence of various values of parameters $b_{1}, b_{2}$ and $c$ on the velocity profile has been seen. It's clear that for constant value of $b_{2}$, there is a direct relation between $b_{1}$ and the velocity.

\section{References}

1. Atangana, A., Baleanu, D.: Numerical solution of a kind of fractional parabolic equations via two difference schemes. Abstract Appl. Anal., vol. 2013, Article ID 828764, p. 8 (2013)

2. Atangana, A., Baleanu, D.: Modelling the advancement of the impurities and the melted oxygen concentration within the scope of fractional calculus. Int. J. Nonlinear Mech. 67, 278-284 (2014)

3. Hayat, T., Shahzad, F., Ayub, M.: Analytical solution for the steady flow of the third grade fluid in a porous half space. Appl. Math. Model. 31, 2424-2432 (2007)

4. Ahmad, F.: A simple analytical solution for the steady flow of a third grade fluid in a porous half space. Commun. Nonlinear Sci. Numer. Simul. 14, 2848-2852 (2009)

5. Kazem, S., Rad, J.A., Parand, K., Abbasbandy, S.: A new method for solving steady flow of a third-grade fluid in a porous half space based on radial basis functions. Z. Naturforsch. 66a, 591-598 (2011) 
6. Finlayson, B.A.: The Method of Weighted Residuals and Variational Principles. Academic Press, New York (1972)

7. Kazem, S., Shaban, M., Rad, J.A.: Solution of Coupled Burger's equation based on operational matrices of d-dimensional orthogonal functions. Zeitschrift fur Naturforschung 67a, 267-274 (2012)

8. Parand, K., Dehghan, M., Baharifard, F.: Solving a laminar boundary layer equation with the rational gegenbauer functions. Appl. Math. Model. 37, 851-863 (2013)

9. Doha, E., Bhrawy, A.: Efficient spectral-galerkin algorithms for direct solution for second-order differential equations using jacobi polynomials. Numer. Algorithm 42, 137-164 (2006)

10. Shateyi, S., Motsa, S.S., Khan, Y.: A new piecewise spectral homotopy analysisof the Michaelis-Menten enzymatic reactions model. Numer. Algorithm 66, 495-510 (2014)

11. Kazem, S.: An integral operational matrix based on Jacobi polynomials for solving fractional-order differential equations. Appl. Math. Model. 37, 1126-1136 (2013)

12. Kazem, S., Abbasbandy, S., Kumar, S.: Fractional-order Legendre functions for solving fractional-order differential equations. Appl. Math. Model. 37, 5498-5510 (2013)

13. Shaban, M., Kazem, S., Rad, J.: A modification on homotopy analysis method based on Chebyshev operational matrices. Math. Comput. Model. 57, 1227-1239 (2013)

14. Guo, B.Y.: Jacobi approximations in certain Hilbert spaces and their applications to singular differential equations. J. Math. Anal. Appl. 243, 373-408 (2000)

15. Guo, B.Y.: Jacobi spectral approximation and its applications to differential equations on the half line. J. Comput. Math. 18, 95-112 (2000)

16. Christov, C.: A complete orthogonal system of functions in $l^{2}(-\infty, \infty)$ space. SIAM J. Appl. Math. 42, 1337-1344 (1982)

17. Boyd, J.P.: Orthogonal rational functions on a semi-infinite interval. J. Comput. Phys. 70, 63-88 (1987)

18. Boyd, J.P.: Spectral methods using rational basis functions on an infinite interval. J. Comput. Phys. 69, 112-142 (1987)

19. Guo, B.Y., Shen, J., Wang, Z.Q.: A rational approximation and its applications to differential equations on the half line. J. Sci. Comput. 15, 117-147 (2000)

20. Boyd, J.P., Rangan, C., Bucksbaum, P.H.: Pseudospectral methods on a semi-infinite interval with application to the Hydrogen atom: a comparison of the mapped Fourier-sine method with Laguerre series and rational Chebyshev expansions. J. Comput. Phys. 188, 56-74 (2003)

21. Parand, K., Razzaghi, M.: Rational Legendre approximation for solving some physical problems on semi-infinite intervals. Phys. Scr. 69, 353-357 (2004)

22. Parand, K., Delafkar, Z., Rad, J.A., Kazem, S.: Numerical study on wall temperature and surface heat flux natural convection equations arising in porous media by rational Legendre pseudospectral approach. Int. J. Nonlinear Sci. 9, 1-12 (2010)

23. Kazem, S., Shaban, M.: Tau-homotopy analysis method for solving micropolar flow due to a linearly stretching of porous sheet. Commun. Numer. Anal. (2012). doi:10.5899/2012/cna-00114

24. Lanczos, C.: Trigonometric interpolation of empirical and analytical functions. J. Math. Phys. 17, 123-199 (1938)

25. Saadatmandi, A., Dehghan, M.: Numerical solution of a mathematical model for capillary formation in tumor angiogenesis via the Tau method. Commun. Numer. Methods Eng. 24, 1467-1474 (2008)

26. Tajvidi, T., Razzaghi, M., Dehghan, M.: Modified rational Legendre approach to laminar viscous flow over a semi-infinite flat plate. Chaos Soliton Fractals 35, 59-66 (2008)

27. El-Daou, M.K., Al-Matar, N.R.: An improved Tau method for a class of Sturm-Liouville problems. Appl. Math. Comput. 216, 1923-1937 (2010)

28. El-Daou, M.K.: Exponentially weighted Legendre-Gauss Tau methods for linear second-order differential equations. Comput. Math. Appl. 62, 51-64 (2011)

29. Saadatmandi, A., Dehghan, M.: A Tau approach for solution of the space fractional diffusion equation. Comput. Math. Appl. 62, 1135-1142 (2011)

30. Doha, E., Abd-Elhameed, W., Bhrawy, A.: Efficient spectral ultraspherical-Galerkin algorithms for the direct solution of 2nth-order linear differential equations. Appl. Math. Model. 33, 1982-1996 (2009)

31. Stegun, I., Abramowitz, M.: Handbook of Mathematical Functions. Dover, New York (1968)

32. Boyd, J.P.: The optimzation of convergence for Chebyshev polynomial methods in an unbounded domain. J. Comput. Phys. 45, 43-79 (1982)

33. Gradshteyn, I.S., Ryzhik, I.M.: Table of Integrals, Series, and Products. Academic Press, New York (2007)

34. Canuto, C., Hussaini, M., Quarteroni, A., Zang, T.: Spectral Methods in Fluid Dynamics. Prentice-Hall, Englewood Cliffs, NJ (1986) 